\title{
Reply to "Ultrasound evaluation of optic nerve sheath diameter to assess treatment efficacy in pediatric idiopathic intracranial hypertension"
}

\author{
Leman Tekin Orgun $^{1,2} \cdot$ Hatice Tuba Atalay ${ }^{3} \cdot$ Ebru Arhan $^{1} \cdot$ Kursad Aydın $^{1} \cdot$ Ayşe Serdaroğlu ${ }^{1}$
}

Received: 24 February 2020 / Accepted: 6 March 2020 / Published online: 17 March 2020

(C) Springer-Verlag GmbH Germany, part of Springer Nature 2020

\section{Dear Editor:}

We would like to thank you for the opportunity to respond to the issues raised in Vitiello et al.'s letter. We also would like to thank Vitiello et al. [1] for their opinion about our recent article [2] published in Child's Nervous System.

We evaluated the usefulness of optic nerve sheath diameter (ONSD) ultrasound measurement in monitoring the treatment efficacy of pediatric idiopathic intracranial hypertension (IIH) by using B-scan orbital ultrasonography (USG). In the present study, the mean ONSDs in orbital ultrasonography were positively correlated with CSF opening pressure levels and measurement of ONSD in MRI. ONSD in orbital ultrasonography regressed gradually consistent with clinical and radiologic improvement during follow-up. [2]

We agree with you that B-scan orbital USG has some handicaps related to technique, patient, and clinicians, which were mentioned in the discussion section of our text and Vitiello et al.'s letter [3-5]. To avoid these disadvantages, ONSD assessments were performed on axial images, which were recorded $3 \mathrm{~mm}$ posterior to the anterior of the optic nerve head, three measurements were obtained in each eye, and their averages were recorded and the cut-off the ONSD enlargement was considered as ONSD of $\geq 5 \mathrm{~mm}$ in the present study. Additionally, serial orbital USGs were performed to evaluate the follow-up of ONSD decreasing. The baseline and control MRI results were

Leman Tekin Orgun

lemantekin15@yahoo.com

1 Pediatric Neurology Department, Gazi University Faculty of Medicine, Ankara, Turkey

2 Department of Pediatric Neurology, Baskent University Faculty of Medicine, Dr Turgut Noyan Teaching and Medical Research Center, Adana, Turkey

3 Ophthalmology Department, Gazi University Faculty of Medicine, Ankara, Turkey used to compare the signs of increased ICP and measurements of ONSD using orbital USG during the follow-up period. Therefore, we believe that the reliability of the B-scan USG was increased avoiding these disadvantages in our study.

In the pediatric population, especially for young children as in our study population, It may not easy to obtain adequate cooperation to perform A-scan USG and to give the directions during the ultrasonography process as Vitiello has mentioned.

We hope that further prospective studies with cooperative older children might be performed to compare to A-scan and B-scan techniques evaluation specifically in pediatric $\mathrm{IIH}$ patients.

\section{Compliance with ethical standards}

Conflict of interest The authors declare that they have no conflict of interest.

\section{References}

1. Vitiello L, De Bernardo M, Rosa N (2020) Ultrasound evaluation of optic nerve sheath diameter to assess treatment efficacy in pediatric idiopathic intracranial hypertension. Childs Nerv Syst:1-2. https:// doi.org/10.1007/s00381-020-04547-0

2. Tekin Orgun L, Atalay HT, Arhan E, Aydın K, Serdaroglu A (2020) Optic nerve ultrasonography in monitoring treatment efficacy in pediatric idiopathic intracranial hypertension. Childs Nerv Syst:1-9. https://doi.org/10.1007/s00381-019-04497-2

3. Irazuzta JE, Brown ME, Akhtar J (2016) Bedside optic nerve sheath diameter assessment in the identification of increased intracranial pressure in suspected idiopathic intracranial hypertension. Pediatr Neurol 54:35-38

4. Koziarz A, Sen N, Kegel F et al (2017) Optic nerve sheath diameter sonography for the diagnosis of increased intracranial pressure: a systematic review and meta-analysis protocol. BMJ Open 7:e016194

5. De Bernardo M, Marotta G, Rosa N (2018) Sonography of the optic nerve sheath diameter. J Ultrasound Med 37:1845

Publisher's note Springer Nature remains neutral with regard to jurisdictional claims in published maps and institutional affiliations. 